\title{
TAKAYASU'S ARTERITIS ASSOCIATED TO MEMBRANOPROLIFERATIVE GLOMERULONEPHRITIS: A CASE REPORT
}

\begin{abstract}
Luiza Lorenzoni Grillo, Debora Marques Veghini1,*, Anna Carolina Simões Moulin, Bárbara Oliveira Souza ${ }^{1}$, Ketty Lysie Libardi Lira Machado ${ }^{1}$, Raiza Cansian Tuao ${ }^{1}$, Barbara Ferraço Dalmaso ${ }^{1}$, Gustavo Pinto de Oliveira Gomes ${ }^{1}$, Gabriel Smith Sobral Vieira ${ }^{1}$, Beatriz Paoli Thompson ${ }^{1}$, Karen Evelin Monlevade Lança ${ }^{1}$, Ruben Horst Duque ${ }^{1}$, Valeria Valim ${ }^{1}$
\end{abstract}

1.Universidade Federal do Espírito Santo, Vitória (ES), Brazil.

*Corresponding author: debora.veghini@hotmail.com

\section{BACKGROUND}

Takayasu's arteritis (TA) is an autoimmune vasculitis that affects the great vessels, mainly the aorta and its great branches. The kidneys are usually affected by narrowing of the renal arteries, a situation that occurs in more than $50 \%$ of the cases of the disease. However, the association of TA with other kidney injuries is still not well documented. The aim of this study was to describe a case of TA associated with membranoproliferative glomerulonephritis (MPGN), a rare condition.

\section{CASE REPORT}

Female, 19 years old, no prior comorbidities, diagnosed with TA five months ago due to the presence of lesions identified on chest and abdomen angiography (circumferential wall thickening of the descending aorta and occlusion of the proximal and middle segments of the left subclavian artery), without involvement of renal arteries, difference in palpation of radial pulses and severe headache. Due to history of precordial pain on medium efforts and edema in the lower limbs, laboratory tests were requested, highlight changes in the values of urea ( $48 \mathrm{mg} / \mathrm{dL})$, creatinine $(2.83 \mathrm{mg} / \mathrm{dL})$, potassium $(5.17 \mathrm{mg} / \mathrm{dL}$ ) and $24 \mathrm{~h}$ proteinuria $(404 \mathrm{mg} / \mathrm{dL})$, raising the suspicion of renal dysfunction. During hospitalization, urinalysis showed albuminuria ( $150 \mathrm{mg} / \mathrm{dL})$, glycosuria (100 mg/dL), leukocyturia (10 leukocytes/field), hyaline, granular, waxes and leukocytes casts. Also, Doppler ultrasound in the aorta and renal arteries revealed no deviations from normality. Based on these results, hemodialysis was performed, culminating in a progressive improvement in the complaints. Additionally, a biopsy revealed findings consistent with MPGN with areas of segmental sclerosis. Immunofluorescence reported the absence of immunoglobulin deposits and complement fractions. Thus, it was necessary to maintain hemodialysis three times a week. Also, pulse therapy was started with methylprednisolone and cyclophosphamide, followed by monthly pulses.

\section{CONCLUSION}

In the case reported, the patient, previously diagnosed with TA, evolved with kidney damage, probably related to the underlying disease. Takayasu's arteritis causes kidney damage mainly due to the narrowing of the renal arteries, but the association with MPGN is rare. In addition, TA has a poorly known pathogenesis, presenting an immunological component, with an important participation of T lymphocytes, as well as MPGN, caused by the deposition of immune complexes. Although it is not possible to say with certainty the correlation of these two conditions, pathogenesis is believed to be similar.

\section{KEYWORDS}

Takayasu’s arteritis, Glomerulonephritis, Vasculitis, Renal injury. 